\title{
Minimum-Cost Optical Amplifier Placement in Metro Networks
}

\author{
Memedhe Ibrahimi, Omran Ayoub, Francesco Musumeci, Oleg Karandin, Andrea Castoldi, Rosanna \\ Pastorelli, Massimo Tornatore
}

\begin{abstract}
Emerging 5G services are revolutionizing the way operators manage and optimize their optical metro networks, and the metro network design process must be rethought accordingly. In particular, minimizing network cost is crucial to curb operators' investment. Taking advantage of relatively-short distances in metro networks, operators have the opportunity to optimize the placement of optical amplifiers (OAs) with the goal of minimizing amplifiers' cost (and hence decrease network cost) without significantly affecting the quality of transmitted optical signals. Minimizing OA cost translates not only in minimizing the cost of equipment (i.e., boosters, pre-amplifiers and inline amplifiers), but also in minimizing deployment and maintenance costs of active amplifier sites. In this paper, we propose a heuristic algorithm for OA placement and for the Routing and Spectrum Assignment (RSA) in metro networks, with the objective of minimizing the total cost of OAs while guaranteeing sufficient optical signal-to-noise ratio (OSNR) of established lightpaths. In our approach, we consider different cost for the deployed OAs, according to their location and type, i.e., inline amplifiers (ILAs), boosters and pre-amplifiers, and compare our optimized placement against benchmark strategies where OAs are predeployed at network nodes and at a fixed distance one from the other along optical fiber links. We also evaluate the impact of different routing strategies on the total cost and utilized spectrum. Simulative results, performed over realistic metro network topologies, show that our strategy provides up to $47 \%$ OAs cost savings while satisfying minimum OSNR constraints.
\end{abstract}

Index Terms - Cross-layer network optimization, Metro Networks

\section{INTRODUCTION}

$\mathrm{O}$ ptical metro networks must be designed keeping in mind the extensive demand for capacity required to support incoming $5 \mathrm{G}$ communications. This capacity increase must be guaranteed while keeping capital expenditures (Capex) and operational expenditure (Opex) under control.

Several cost-effective solutions such as, e.g., filterless networking [1-2] and wavelength-interleaved networks [3], are being investigated to replace costly state-of-the-art wavelengthswitched architectures in metro. These solutions, however, are still under investigation, and cannot be used to satisfy the current need to reduce costs in the medium-short term. An

This paper is submitted for review on November 22, 2019. This work is supported by a sponsored research agreement contract with SM-Optics. (Corresponding author: Memedhe Ibrahimi)

M. Ibrahimi, O.Ayoub, F. Musumeci, O. Karandin and M. Tornatore are with Politecnico di Milano, via Giuseppe Ponzio 34, 20133 Milano, Italy (e- alternative and less-disruptive strategy to minimize costs in metro networks consists in reducing the amount of expensive optical devices as well as the number of active sites in the network. Considering the relatively short distances in metro networks, operators can achieve this goal by optimizing the number, location and type of optical amplifiers (OAs) in egress of network nodes, i.e., booster amplifiers (boosters), in ingress of network nodes, i.e., pre-amplifiers (pre-amps), and those located along fiber links, i.e., in-line amplifiers (ILAs), while guaranteeing sufficient optical signal-to-noise ratio (OSNR) for the established lightpaths. Note that, decreasing the amplifiers' costs in a metro network does not only mean less equipment cost (less amplifiers), but, even more importantly, also cutting costs by reducing the number of active-node locations in metro areas (e.g., amplifiers huts).

Overall, the optimization problem to be addressed to return a feasible design with minimal amplifier cost can be summarized as quality of transmission (QoT)-aware routing and spectrum allocation with amplifier placement (RSA-AP). To solve the RSA-AP problem, we develop a heuristic approach which performs two main tasks: 1) RSA of traffic demands and 2) Placement of OAs. Since amplifier placement has a strong impact on the performance of optical signal transmission, an accurate physical layer modelling to capture the impact of amplifier placement on the Quality-of-Transmission, i.e., on the OSNR of lightpaths is needed. For this aim, we make use of well-known analytical non-linear propagation models [4] (detailed description of physical model is presented in Sec. II.A) and incorporate it in the proposed heuristic approach.

\section{A. Related Work}

The problem of amplifier placement with the objective of minimizing cost of amplifiers has received little attention in literature, as minimizing amplifiers cost in long-distance regional/backbone networks is not expected to lead to significant cost savings. In fact, signal amplification and regeneration in long-distance backbone networks is most often required, hence the placement of ILAs, boosters and preamplifiers cannot be avoided, making the associated RSA-AP problem ineffective in cutting costs in long distance networks. Only some recent work on amplifier placement refers to long-

mails:memedhe.ibrahimi@polimi.it;omran.ayoub@polimi.it;francesco.musum eci@polimi.it; oleg.karandin@mail.polimi.it; massimo.tornatore@ polimi.it).

A. Castoldi and R. Pastorelli are with SM-Optics, via P.Castaldi, 8, 20124, Milano, Italy(e-mails:andrea.castoldi@sm-optics.com; rosanna.pastorelli@smoptics.com). 
haul communications where the placement of amplifiers along with regenerators is considered. Ref. [5] proposes a heuristic method to optimize the placement of hybrid Raman/EDFA amplifiers in mesh optical networks. It determines the placement of Raman amplification such that the number of optical regenerators is minimized. Ref. [6-7] consider the selective upgrade of line optical amplifiers to hybrid Raman/Erbium amplification in order to reduce the number of optical regenerators. Ref. [8] studies the problem of the upgrade of optical line amplifiers with Raman amplification (RA) modules and proposes three strategies for equipment upgrade. Conversely, in metro, the relatively high number of nodes (in this paper assumed to be Reconfigurable Optical Add-Drop Multiplexers - ROADMs) and short fibers make the problem tempting for operators. Seminal works in [9-10] investigated amplifier placement in metro networks providing mixed integer linear formulation with the objective of minimizing the number of amplifiers, but neglecting the impact of amplified spontaneous emission (ASE) noise introduced by amplifiers, which has a major impact on the OSNR of signals in the network. In our work, we consider the impact of ASE noise in signal propagation as well as the non-linearities due to signal propagation by using fiber non-linear propagation models which in turn do not enable an ILP formulation for this problem. We extend the preliminary analysis reported in [11] and investigate the placement of boosters, pre-amps and ILAs in metro networks employing coherent detection and uncompensated transmission techniques which allow us to consider a well-established and computationally-efficient GNModel [4] for non-linear propagation.

\section{B. Paper Contribution and Organization}

The main contributions of this paper are as follows: 1) we formally define the RSA-AP problem in metro networks; 2 ) we propose a novel heuristic (the MinOA algorithm) approach to solve the RSA-AP problem, which minimizes the overall cost of OAs in the network while guaranteeing QoT of established lightpaths; 3 ) we discuss a model to set the gain of amplifiers in the network with focus on the case when boosters and preamps are absent at network nodes; 4) through a simulative study, we evaluate the impact of routing and optimized placement of boosters, pre-amps and ILAs on both the OSNR of lightpaths and the overall spectrum occupied in realistic metro topologies.

The rest of the paper is organized as follows. Section II introduces the OA placement problem and describes the physical layer model considered in our analysis. Section III states the RSA-AP problem and describes the heuristic algorithm proposed to solve it. Section IV presents numerical results and Sec. V draws paper conclusion.

\section{BACKGROUND}

\section{A. Physical layer description}

We consider the Gaussian Noise propagation model (GNmodel). The GN-model allows to predict the performance of an uncompensated optical link by accurately estimating the nonlinear interference (NLI) introduced by the signal propagation in the optical fiber.

The value of the optical signal-to-noise ratio received at the receiver for an optical lightpath is defined as the total OSNR (OSNR $\left.{ }_{T O T}\right)$. It accounts for the linear OSNR contribution, i.e., $\mathrm{OSNR}_{\mathrm{ASE}}$, and the non-linear contribution, i.e., $\mathrm{OSNR}_{\mathrm{NL}}$, and for an optical lightpath traversing $\mathrm{k}$ optical spans, it is calculated as follows:

$$
\frac{1}{O S N R_{T O T}}=\sum_{k=1}^{N_{S p a n s}} \frac{1}{O S N R_{k, A S E}}+\sum_{k=1}^{N_{\text {spans }}} \frac{1}{O S N R_{k, N L}}
$$

Since the linear OSNR $\left(O S N R_{A S E}\right)$ contribution is due to the amplifiers in the network, care must be taken when deploying amplifiers and setting their gain so that signal quality is ensured and so that the ASE noise introduced by the OAs does not cause the signal to deteriorate below a certain quality level. In addition, the non-linear OSNR $\left(O S N R_{N L}\right)$ depends on the fiber characteristics (local chromatic dispersion, refractive index, attenuation coefficient, span length) and on the traffic matrix. The linear OSNR and the nonlinear OSNR of a lightpath in the $k^{\text {th }}$ span are defined in [12] as follows:

$$
O S N R_{k, A S E}=\frac{P_{k}}{h v B_{n} G_{k} F_{k}} \quad \text { (2) and } O S N R_{k, N L}=\frac{1}{B_{n} \eta_{k} P_{k}^{2}}
$$

where $P_{k}$ is the channel power, $B_{n}$ is the noise reference bandwidth, $h$ is the Planck's constant, $v$ is the center propagation frequency, $G_{k}$ is the gain and $F_{k}$ is the noise figure of the $\mathrm{k}^{\text {th }}$ amplifier, $\eta_{k}$ is the non-linear efficiency (calculated as given in [4]).

In our analysis we consider Standard Single-Mode Fiber (SSMF) links with an attenuation coefficient of $0.22 \mathrm{~dB} / \mathrm{km}$ and $0.03 \mathrm{~dB} / \mathrm{km}$ extra attenuation due to splice losses which amounts to $0.25 \mathrm{~dB} / \mathrm{km}$ propagation loss, dispersion coefficient of 21 $\mathrm{ps}^{2} / \mathrm{km}$ and fiber non-linearity coefficient $1.3 \mathrm{~W}^{-1} \mathrm{~km}^{-1}$. We compute lightpaths OSNR through the GN-Model assuming full spectral load and links operating at their optimal power, as prescribed by the Locally-Optimized-Globally-Optimized (LOGO) strategy [12-13]. According to the LOGO approach, $O S N R_{T O T}$ can be maximized by finding the optimal value of the launched power in each span. For a span $k$, characterized by a span loss $A_{k, \text { span }}$, the non-linearity efficiency $\eta_{k}$ and noise figure of the OA at the end of the $k^{\text {th }}$ span $F_{k}$, the optimal launched power is defined as follows:

$$
P_{k, \text { opt }}=\left(\frac{h v A_{k, s p a n} F_{k}}{2 \eta_{k}}\right)^{1 / 3}
$$

To determine lightpaths feasibility, the total OSNR $\left(O S N R_{T O T}\right)$ of a lightpath is required to be above the OSNR threshold, i.e., the minimum required OSNR for a lightpath. Note that the OSNR threshold is given as the required OSNR considered in the back-to-back configuration $\left(O S N R_{B 2 B}\right)$ [14]. In order to account for the impact of effects such as aging, optical filtering and power ripple, we consider a conservative system margin (i.e., variable Margin) of $2 \mathrm{~dB}$. Therefore, a lightpath is defined to be feasible if its total OSNR $\left(O S N R_{T O T}\right)$ is greater than the threshold, as given in (5):

$$
\operatorname{OSNR}_{T O T}>\operatorname{OSNR}_{B 2 B}+\text { Margin }
$$

In addition to OSNR, to determine lightpath feasibility, also 
the received power $\left(P_{R E C}\right)$ needs to be taken into consideration. $\mathrm{P}_{\mathrm{REC}}$ is computed as follows:

$$
P_{R E C}=P_{T X}-\sum \text { Loss }+\sum \text { Gain } \quad[\mathrm{dBm}]
$$

where $P_{T X}$ is the channel power launched at the source of the lightpath, sum of Loss accounts for all losses along the path, i.e., due to propagation loss, node loss and variable optical attenuators (VOAs) and the sum of Gain accounts for the gain of all amplifiers deployed along the path. We assume the total amount of loss of an express node to sum up to $17 \mathrm{~dB}$ due to splitters, combiners, VOAs and wavelength selective switches (WSSs) in the node while total loss of source and destination nodes is assumed to be half the loss of an express node (i.e., their joint loss contribution is equal to an express node loss).

\section{B. Optical Amplifier placement}

We consider the placement of amplifiers in three different locations: 1) at the ingress of a node, referred to as pre-amp, 2) at the egress of a node, referred to as booster and 3) along the fiber, referred to as ILA. Moreover, we consider two different types of amplifiers to be deployed in these locations. These two types of amplifiers differ in their physical characteristics, i.e., in terms of their gain and noise figure range (see Table I).

Table I. Optical Amplifier types and their characteristics

\begin{tabular}{|c|c|c|}
\hline OA type & Gain [dB] & Noise Figure [dB] \\
\hline Booster & $10-20$ & $12-6$ \\
\hline Pre-amp & $18-32$ & $10.5-6.2$ \\
\hline
\end{tabular}

The relation between noise figure and gain of an amplifier is the following: $F=a+\frac{b}{G-1}$, given in linear units. The values of $a$ and $b$ for booster OA type are: $\mathrm{a}=2.793$ and $\mathrm{b}=117.513$ and for pre-amp OA type are: $a=3.88$ and $b=455.814$. In the figure below, we show the relation between gain and noise figure in $\mathrm{dB}$ units for both types of amplifiers we consider, booster and pre-amp OA type.

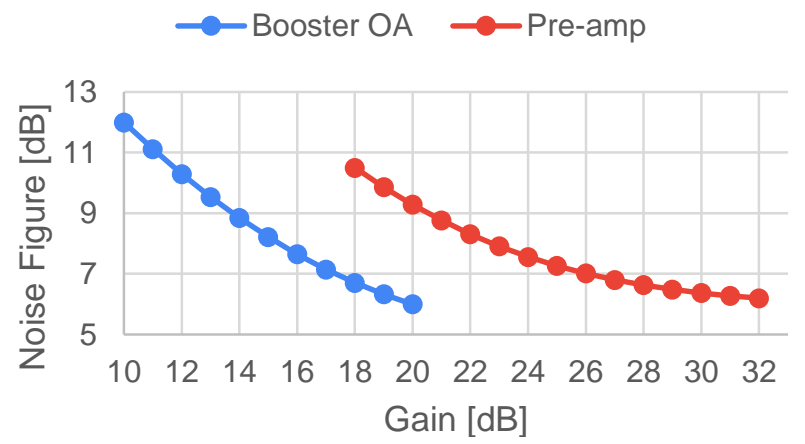

Figure 1. Noise Figure $(d B)$ as a function of Gain $(d B)$ for booster amplifiers (booster $O A$ ) and pre-amplifiers (pre-amps). Note that the gain range differs for each of amplifiers type. The curves show the relation between gain and noise figure for booster and pre-amp OA types

Considering that pre-amplifier type has higher gain values, we assume that it has a higher cost than the booster OA type. Common practice constrains the deployment of first amplifier

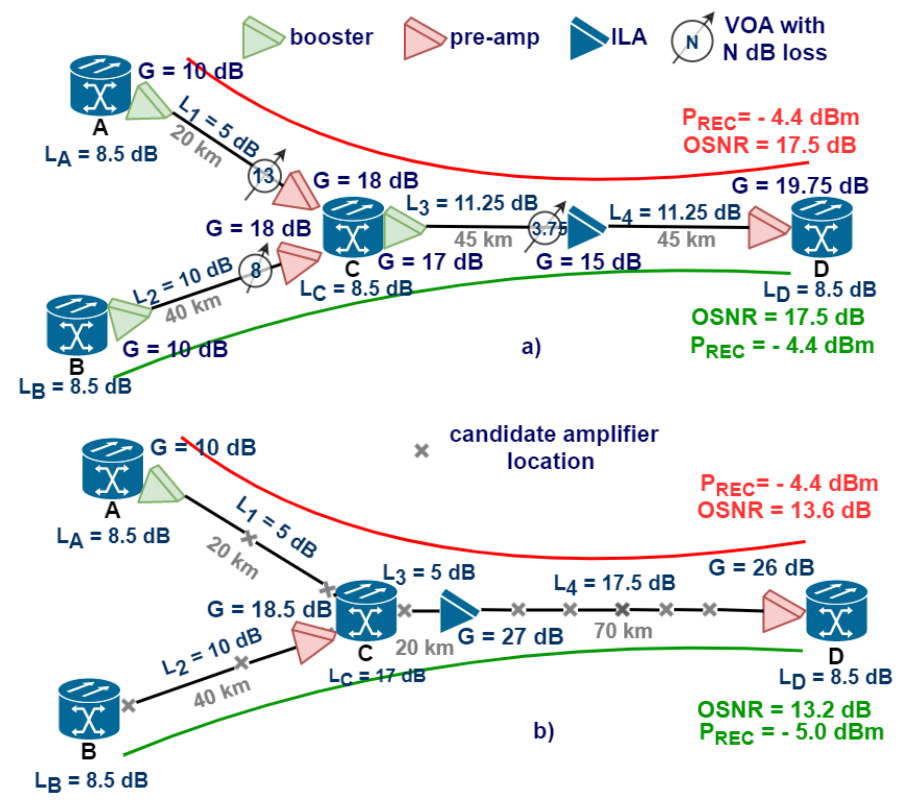

Figure 2. Example of OA placement a) baseline approach and b) optimized placement

type at egress of network nodes (therefore it is usually referred to as booster), and the deployment of second amplifier type at ingress of network nodes (therefore it is usually referred to as pre-amp). In our work, however, we do not restrict the deployment of any specific type of amplifiers at any location in the network. Instead, we allow the deployment of any amplifier type in any candidate amplifier locations, i.e., along the fiber or at ingress or egress of nodes, to evaluate possible cost savings with respect to a common practice case, where specific amplifier types are designed constrained to specific locations. As a consequence, the choice of the amplifier to be placed in a given location $x$ depends on the desired gain for that location, i.e., on the optical span it terminates and its characteristics. In other words, if the desired gain is in the range (10-20) dB we assume the placement of booster amplifier type, while, if the desired gain ranges between (20-32) dB, we assume the placement of pre-amplifier type. In case there is a span whose loss is less than the minimal gain of the placed OA, e.g., span loss is $7 \mathrm{~dB}$ and minimal gain is $10 \mathrm{~dB}$, we enforce the constraint of the minimal OA gain and consider additional attenuation ( $3 \mathrm{~dB}$ attenuation in this case) performed by a VOA, so that the span loss equals the OA gain and it is compensated.

Let us consider the simple example in Fig. 2, which illustrates the principle of OA placement and its effect in the lightpaths on the network. Fig. 2(a) shows a traditional OA placement, whereas Fig. 2(b) shows an optimized OA placement.

We consider two lightpaths originating at nodes A and B with the same destination node $\mathrm{D}$. In case a), boosters and pre-amps are placed at each node and also an ILA placed in link C-D. Such deployment ensures that OSNR and $\mathrm{P}_{\mathrm{REC}}$ requirements are ensured for both lightpaths, as shown in the figure. In case b) OAs can be placed in any of the candidate amplifier locations indicated by a " $x$ ". The optimized OA placement returns fewer OAs yet satisfying the QoT requirements for both lightpaths. Specifically, we save a pre-amp and two boosters in comparison 
to the case in Fig. 2(a).

The example in Fig. 2(b) shows that there is no pre-amp at node ingress in link A-C and no booster at egress of nodes B and $\mathrm{C}$, therefore the ILA placed just after node $\mathrm{C}$ will set its gain to compensate for both the node loss and propagation loss. Please note that in a case of a ROADM node without booster and pre-amp, the amplifier placed after the node may terminate different spans for different lightpaths. This determines also the power level of the lightpaths passing through the node. Since ROADM nodes are equipped with VOAs and perform power equalization, two lightpaths reaching the node with different power levels will exit the node with the same power according to the lightpath(s) with the lowest power level. In fact, since both lightpaths pass through node $\mathrm{C}$, the VOA at the node will allow the lightpaths to have the same power after node $C^{1}$. The ILA in link C-D terminates two different spans for both lightpaths and thus its gain is set so that it compensates for the span of highest loss (lightpath A-D). Its gain considers the loss in span $\mathrm{L}_{1}, \mathrm{~L}_{3}$ and the loss of node $\mathrm{C}\left(\mathrm{L}_{\mathrm{C}}\right)$ amounting to $27 \mathrm{~dB}$.

Regarding the $\mathrm{P}_{\mathrm{REC}}$ constraint, since the gain of OAs along both paths is set to compensate for the losses in each span they terminate, also the $\mathrm{P}_{\mathrm{REC}}$ constraint is met for both lightpaths ${ }^{2}$. Let us observe the calculation of the received power for lightpath B-D:

$\mathrm{P}_{\mathrm{REC}}=\mathrm{P}_{\mathrm{TX}}-\mathrm{L}_{\mathrm{B}}-\mathrm{L}_{2}+\mathrm{G}-\mathrm{L}_{\mathrm{C}}-\mathrm{VOA}_{\mathrm{C}}-\mathrm{L}_{3}+\mathrm{G}-\mathrm{L}_{4}-\mathrm{L}_{\mathrm{D}}+\mathrm{G}=-5-8.5-$ $10+18.5-17-5-5+27-17.5-8.5+26=-5 \mathrm{dBm}$. Node losses are noted according to node id $\left(\mathrm{L}_{\mathrm{B}}\right.$ is loss due to node $\left.\mathrm{B}\right)$, propagation losses by the span id ( $\mathrm{L}_{2}$ is loss in link $\left.\mathrm{B}-\mathrm{C}\right), \mathrm{VOA}_{\mathrm{C}}$ is the VOA attenuation at node $\mathrm{C}$ and $\mathrm{OA}$ gains as $\mathrm{G}$.

In conclusion, the same principle of OA placement shown in this example can be followed in more complex scenarios and may lead to significant cost savings in terms of placed OAs.

\section{PROPOSED STRATEGY FOR OPTICAL AMPLIFIER PLACEMENT AND ROUTING AND SPECTRUM ASSIGNMENT}

\section{A. Problem Statement}

The problem of RSA-AP placement can be formally stated as: Given a metro network topology, set of traffic demands (characterized by source and destination nodes, bit-rate request and modulation format) and set of candidate locations of optical amplifiers, decide the routing and spectrum allocation to all traffic demands and placement of OAs, constrained by $i$ ) quality-of-transmission constraint (sufficient OSNR and received power) of all traffic demands, ii) spectrum continuity and contiguity constraints, iii) network capacity constraints and iv) amplifiers gain range constraint with the Objective of minimizing the total cost of deployed OAs.

\section{B. RSA-AP heuristic approach}

The flow chart of MinOA heuristic algorithm is shown in Fig. 3. The algorithm is constituted by two main intertwined phases:

\footnotetext{
${ }^{1}$ Note that we model a ROADM node as a fiber span of $68 \mathrm{~km}$ (equivalent to $17 \mathrm{~dB}$ loss). We assume that no non-linear interference (NLI) is generated along the fiber span which mimics the ROADM node. Note also that, in case of no pre-amplifier and booster being located at a node, only the fiber span located before the node is accounted when calculating NLIs (non-linear efficiency parameter $\eta$ ).
}

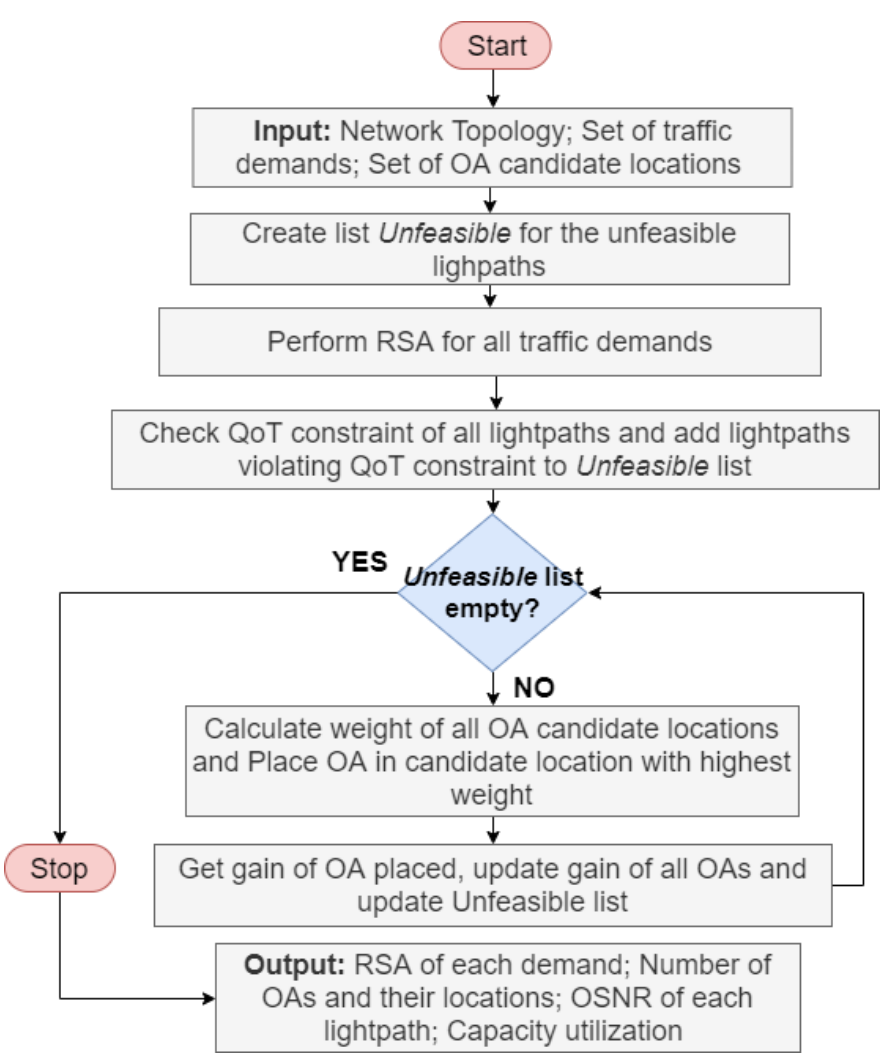

Figure 3. Heuristic flowchart

1) RSA for traffic demands and 2) Optical Amplifier Placement.

\section{1) Routing and Spectrum Allocation}

To perform the routing of lightpaths, we consider two routing algorithms, i) Shortest-Path $(S P)$ routing, which, for each demand, utilizes the path with minimum distance in kilometers, i.e., corresponding to minimizing the fiber propagation loss ${ }^{3}$, and $i$ ) Minimal-Loss $(M L)$ routing, which routes a lightpath on the path with the minimum loss on the end-to-end path, i.e., the sum of node losses and propagation losses minus the sum of the gain of OAs in the path. Fig. 4 shows a ring-link section of a metro network over which we can compare the routing of a

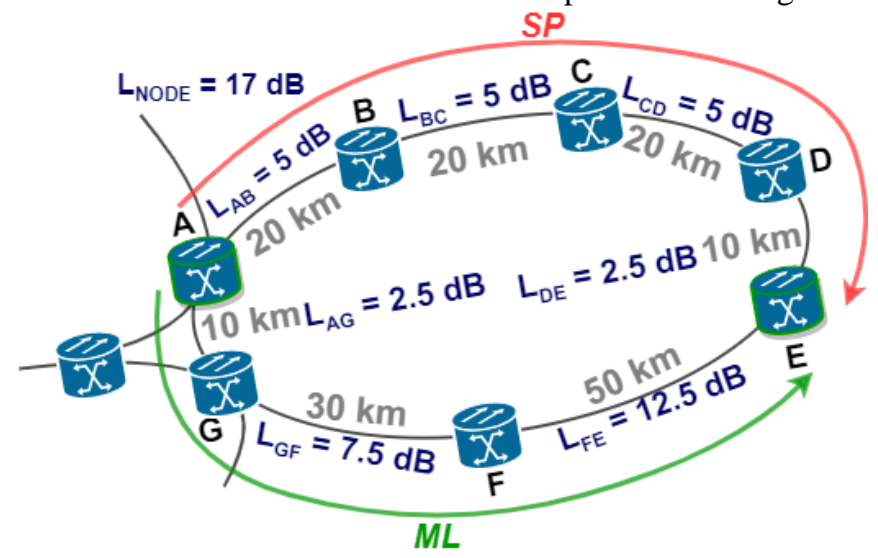

Figure 4. Sample topology used to compare $M L$ vs $S P$ routing

${ }^{2}$ Considering that the two lightpaths have different first spans, their launch power is slightly different and dependent on the span characteristics, as per LOGO principle.

${ }^{3}$ Note that this holds as we assume that all the fibers in the network have the same attenuation coefficient $(0.25 \mathrm{~dB} / \mathrm{km})$. 
lightpath from node $A$ to node $E$, by using the two routing algorithms, $S P$ and $M L$. With $S P$ routing algorithm, the lightpath is assigned to path $A-B-C-D-E$ with a total length of $70 \mathrm{~km}$ (total loss is $85.5 \mathrm{~dB}$ ), while with $M L$ algorithm, the lightpath is assigned the path A-G-F-E, having a total length of $90 \mathrm{~km}$ and total loss of $73.5 \mathrm{~dB}$. As for the spectrum assignment, we consider flex grid spectrum allocation with first-fit allocation policy.

After the RSA of all traffic demands is performed, the OSNR and $P_{R E C}$ values are calculated for all the demands, which are then distinguished between feasible and unfeasible. A demand is considered unfeasible if its OSNR and/or $P_{\text {REC }}$ are below the corresponding threshold values. Unfeasible lightpaths are then inserted in a list called unfeasible lightpaths.

\section{2) Optical Amplifier Placement}

MinOA evaluates placing an $\mathrm{OA}$ in each candidate $\mathrm{OA}$ location considering its impact on the OSNR and $\mathrm{P}_{\mathrm{REC}}$ of the lightpaths traversing the location, as well as the cost of the OA to be deployed at that location, which is determined according to the required OA gain, and hence on the amplifier type.

We determine the weight of each candidate OA location using a "weight function" and, at each iteration, the candidate OA location with highest weight is selected and an OA is deployed there. Considering the importance of the weight function in our approach, we modelled several weight functions and compared their performance over different network instances and then considered the weight function which led to the best performance.

The weight of a candidate location $j, w_{j}$, is given by the sum of the OSNR improvements plus the sum of a feasibility parameter for all unfeasible lightpaths passing through that location, all divided by the cost of the OA considered for location $j$, as in (7).

$$
w_{j}=\frac{\sum_{i \in I}\left(F_{i}+\Delta O S N R_{i}\right)}{c_{j}}
$$

where, i) $\triangle O S N R_{i}$ is the OSNR improvement for unfeasible lightpath $i$ achieved thanks to the placement of OA at location $j$ taking into account the current OSNR value $\left(O S N R_{i, n o w}\right)$ and the OSNR in the previous iteration $\left(O S N R_{i, p r e v}\right)$ and given as:

$$
\Delta O S N R_{i}=O S N R_{i, \text { now }}-O S N R_{i, p r e v} \quad[\mathrm{~dB}]
$$

ii) The feasibility parameter for lightpath $i, F_{i}$, depends on the OSNR of lightpath $i\left(O S N R_{i}\right)$ and the received power of lightpath $i\left(P_{R E C, i}\right)$ in current iteration and is defined as:

$$
F_{i}=\left\{\begin{array}{l}
2, \text { if } O S N R_{i}>\text { threshold and } P_{R E C, i}>\text { threshold } \\
1, \quad \text { otherwise }
\end{array}\right.
$$

If an unfeasible lightpath $i$ which passes through location $j$ will become feasible $\left(\mathrm{OSNR}_{\mathrm{i}}>\right.$ threshold and $\mathrm{P}_{\mathrm{RECi}}>$ threshold) by

\footnotetext{
${ }^{4}$ Note that, as in our heuristic the routing is performed in the first step and does not change with the execution of the OA placement, for all the strategies adopting $S P$, routing of lightpaths does not depend on the initial OA deployment. Conversely, the initial OA deployment affects the routing in case
}

placing an $\mathrm{OA}$ in $j$, its feasibility parameter is considered to be twice compared to the case when it remains unfeasible.

iii) $C_{j}$ is the cost of the OA type which depends on the gain, noise figure and location placed, as detailed later in Sec. IV.

After the placement of a new amplifier at each iteration, the optical spans in the network and the gain and noise figure of all the existing amplifiers are updated accordingly, i.e., considering the optimal input power at each span as provided by the LOGO approach, and the OSNR and $\mathrm{P}_{\mathrm{REC}}$ of all lightpaths are calculated and the lightpaths which now meet the QoT constraint are removed from the list of unfeasible lightpaths. Finally, minOA stops when no more unfeasible lighpaths exist in the network, i.e., when all the lightpaths in the network meet their QoT requirements. If unfeasible lightpaths are still present in the network, minOA starts a new iteration to place a new amplifier until no more unfeasible lightpaths are present in the network or when no other OA candidate location is available.

\section{NUMERICAL RESULTS}

We numerically evaluate the MinOA algorithm, by considering three scenarios which differ in the initial deployment of OAs, i.e.: 1) MinOA, representing the proposed approach where we assume a green-field scenario where no amplifiers are deployed at the beginning in the network, 2) MinOA-bst, where we assume an initial deployment with boosters in all network nodes, and 3) MinOA-pre, assuming an initial deployment with pre-amplifiers in all network nodes. For each case we consider the two routing algorithms described previously, $M L$ and $S P$ resulting in 6 cases ( $S P$-MinOA, $S P$ MinOA-pre, SP-MinOA-bst, $M L$-MinOA, $M L$-MinOA-pre, $M L$ MinOA-bst). Furthermore, we compare the 6 MinOA versions against two baseline amplifier placement approaches, where we assume boosters and pre-amps deployed at all network nodes and assuming ML routing (ML-Baseline) and SP routing (SPBaseline). Baseline OA deployment assumes that the booster amplifier is set to compensate for the node loss whereas the preamp for the span it terminates. ILA amplifiers are placed approximately every $60 \mathrm{~km}$ (corresponding to a gain of $15 \mathrm{~dB}$ ). Note that this assumption might be slightly adjusted, depending on the length of the fiber. If the length of the link is longer than $60 \mathrm{~km}$, an ILA is placed in the middle of the link. For example, if the length of the link is $80 \mathrm{~km}$, an ILA is placed in the middle of the link, e.g., at $40 \mathrm{~km}$. However, if the length of the link is $120 \mathrm{~km}$ or longer, ILAs are placed approximately every $60 \mathrm{~km}$. Overall, 6 different scenarios of MinOA and 2 versions of baseline approach are considered in our study, as summarized in Table $\mathrm{II}^{4}$.

Finally, in our results we consider two cases regarding the deployment of amplifiers in candidate locations: 1) locationunconstrained, where any type of amplifier can be deployed in any location, and 2) location-constrained, where the type of amplifier deployed in a specific location has to correspond to location characteristics. The cost of each amplifier type is

$M L$ is adopted as, besides the loss contributions between source and destination nodes, when calculating loss along a path $M L$ routing considers also the gain of pre-deployed amplifiers. 
according to field observations and reflects the characteristics (gain and noise figure) of OAs. We assume the cost of a booster is 1 cost unit $(\mathrm{cu})$ while the cost of a pre-amp is assumed equal to $1.2 \mathrm{cu}$. If deployed as an ILA, the cost of an amplifier assumes 0.8 additional $\mathrm{cu}$ accounting for the additional site cost, and so it is $1.8 \mathrm{cu}$ if a booster type is deployed and $2.0 \mathrm{cu}$ if a pre-amp type is deployed.

Table II. Different OA placement scenarios

\begin{tabular}{|c|c|c|c|}
\hline Approach & Routing & Placement & Pre-deployed \\
\hline SP-MinOA & $S P$ & MinOA & None \\
\hline SP-MinOA-bst & $S P$ & MinOA & Boosters \\
\hline SP-MinOA-pre & $S P$ & MinOA & Pre-amps \\
\hline ML-MinOA & $M L$ & MinOA & None \\
\hline ML-MinOA-bst & $M L$ & MinOA & Boosters \\
\hline ML-MinOA-pre & $M L$ & MinOA & Pre-amps \\
\hline SP-Baseline & $S P$ & Baseline & \multirow{2}{*}{$\begin{array}{c}\text { Boosters, pre- } \\
\text { amps and ILAs }\end{array}$} \\
\hline ML-Baseline & $M L$ & Baseline &
\end{tabular}

\section{A. Validation of the heuristic}

In order to validate the effectiveness of MinOA, we compare its performance with that of brute force (Brute) approach. Note that a comparison with an optimal ILP-based placement is not possible due to the non-linear nature of the problem. We considered several case studies varying network topology, length of links and number of candidate locations. In Table III we report the results for MinOA and Brute for one topology composed by 8 nodes and 16 links. We increase the length of the links and consequently increase the number of candidate locations. Fig. 5 shows the topology considered for the validation of the heuristic.

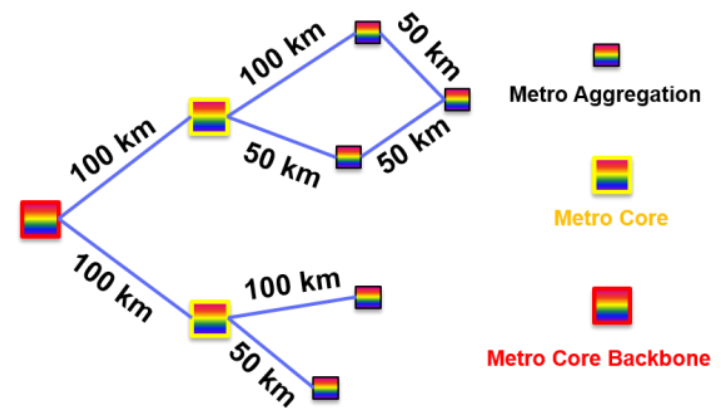

Figure 5. A sample section of a metro network topology (the case study with $\mathbf{4 0}$ amplifier candidate locations)

We consider a full-mesh traffic matrix with $100 \mathrm{Gbps}$ and DPQPSK demands. Results show that the overall cost of amplifiers considering MinOA is comparable to that of Brute, as the cost difference is always below 7\%. Even if for such a small network instance, Brute has a computational time in the order of days, whereas MinOA provides a solution in order of minutes.

Table III. Total cost of amplifiers ( $c u$ ) for MinOA and Brute

\begin{tabular}{|l|c|c|c|c|c|}
\hline Nr. of candidate loc. & $\mathbf{3 2}$ & $\mathbf{3 4}$ & $\mathbf{3 6}$ & $\mathbf{3 8}$ & $\mathbf{4 0}$ \\
\hline MinOA & 34.2 & 37.8 & 42.4 & 45 & 48.6 \\
\hline Brute-force & 32.2 & 35.8 & 40.4 & 42 & 45.6 \\
\hline Difference [\%] & 5.8 & 5.2 & 4.7 & 6.7 & 6.2 \\
\hline
\end{tabular}

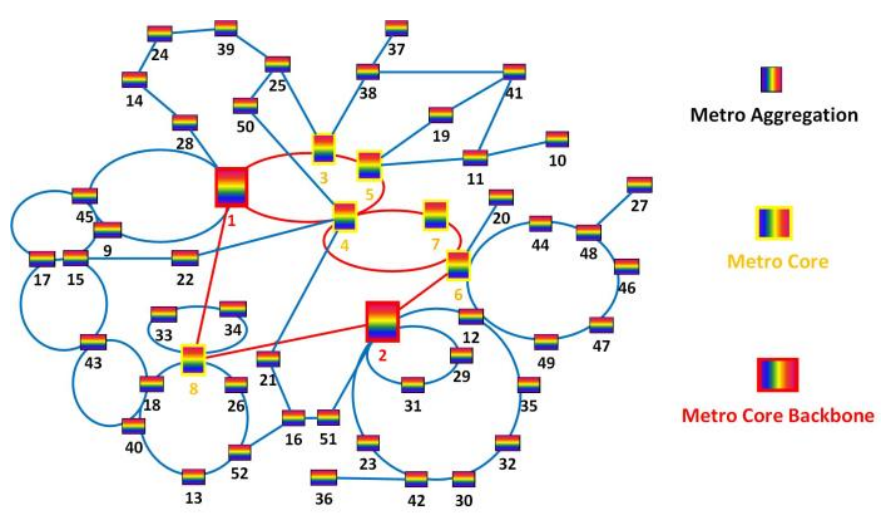

Figure 6. Schematic representation of 52Node-Metro network topology

\section{B. Numerical results and discussion}

We now consider two realistic metro topologies, referred to as 52Node-Metro (Fig. 6) and 159Node-Metro (Fig. 8). Characteristics of the two network topologies are shown in Table IV. The topologies include three types of network nodes, core-backbone, metro-core and metro-aggregation nodes.

Table IV. Characteristics of metro topologies considered

\begin{tabular}{|l|c|c|}
\hline \multicolumn{1}{|c|}{ Network } & 52Node-Metro & 159Node-Metro \\
\hline Nodes & 52 & 159 \\
\hline Links & 144 & 438 \\
\hline Diameter & $140 \mathrm{~km}$ & $230 \mathrm{~km}$ \\
\hline Max/min length & $200 / 1 \mathrm{~km}$ & $240 / 1 \mathrm{~km}$ \\
\hline \# Cand. OA loc. & 402 & 1144 \\
\hline
\end{tabular}

We consider full-mesh bi-directional traffic demands between metro-core and metro-core backbone nodes, and bi-directional traffic demands between metro-aggregation nodes and nearest metro-core nodes, simulating traffic exchanged between regional data centres and end-users. We consider frequency slot units (FSUs) of a granularity of $12.5 \mathrm{GHz}$ and a $5 \mathrm{THz}$ spectrum capacity, i.e., equivalent to 400 FSUs per link. We consider 100 Gbps and 200 Gbps traffic with DP-QPSK modulation format. Specifically, the traffic demands generated between metro-core and metro-core backbone nodes are $200 \mathrm{Gbps}$ bitrates whereas the traffic demands between metro-aggregation nodes and nearest metro-core nodes are $100 \mathrm{Gbps}$. Each 100 -Gbps traffic demand occupies a channel of $37.5 \mathrm{GHz}$, i.e., 3 FSUs, while each 200-Gbps traffic demand occupies a channel of $75 \mathrm{GHz}$, i.e., 6 FSUs.

Note that the feasibility of each demand depends on guaranteeing an OSNR threshold of $\mathbf{1 3} \mathbf{~ d B}$ and minimum received power $P_{R E C}$ of $\mathbf{- 1 8} \mathbf{~ d B m}$. Fig. 7 shows the overall cost of OAs deployed for each OA deployment strategy including baseline approach. Moreover, we show in Table $\mathrm{V}$ the minimum and average OSNR values in each of the cases.

\section{1) MinOA vs. Baseline}

Comparing the various versions of $\mathrm{MinOA}$ to the baselines, we observe that MinOA achieves cost savings between 26\% and $30 \%$ in the location-unconstrained case, and between $30 \%$ and $35 \%$ in the location-constrained case. 


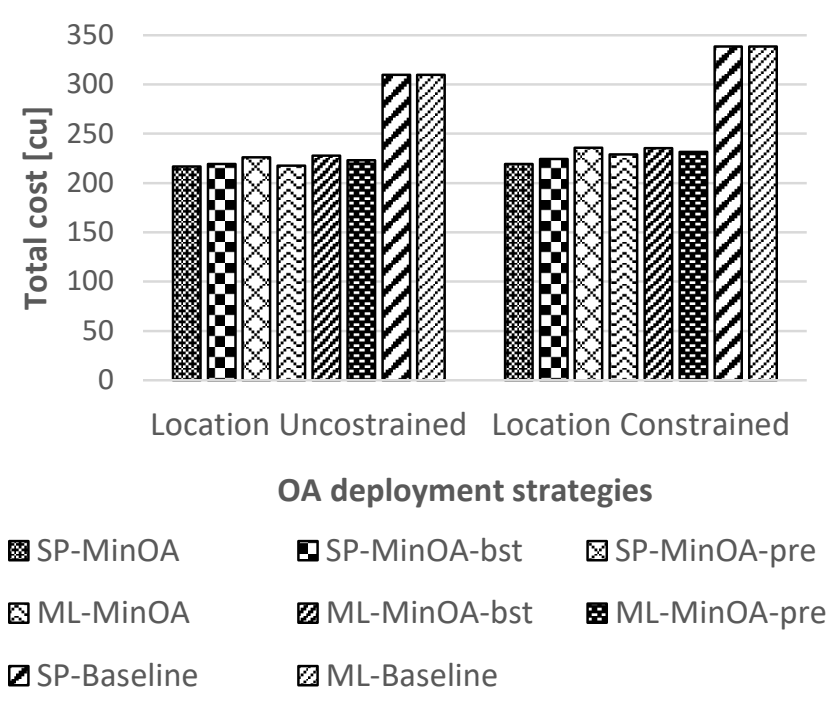

Figure 7. Total cost of OAs deployed for 52Node-Metro network

Table V. OSNR characteristics in dB for the different OA deployment strategies considered

\begin{tabular}{|c|c|c|}
\hline \multirow{2}{*}{ Strategy } & $\begin{array}{c}\text { Location } \\
\text { Unconstrained }\end{array}$ & $\begin{array}{c}\text { Location } \\
\text { Constrained }\end{array}$ \\
\cline { 2 - 3 } & minOSNR (avgOSNR) & minOSNR (avgOSNR) \\
\hline SP-MinOA & $13.67(20.95)$ & $13.38(20.02)$ \\
\hline SP-MinOA-pre & $13.10(18.75)$ & $13.03(17.37)$ \\
\hline SP-MinOA-bst & $14.48(21.02)$ & $13.99(20.30)$ \\
\hline ML-MinOA & $14.70(21.26)$ & $14.77(20.53)$ \\
\hline ML-MinOA-pre & $14.30(18.91)$ & $14.20(17.57)$ \\
\hline ML-MinOA-bst & $14.16(21.27)$ & $14.00(20.66)$ \\
\hline SP-Baseline & $13.82(20.81)$ & $13.01(17.98)$ \\
\hline ML-Baseline & $13.19(20.74)$ & $13.00(18.23)$ \\
\hline
\end{tabular}

This is because $\mathrm{MinOA}$, at each iteration, places amplifiers in locations which mostly improve OSNR and $\mathrm{P}_{\mathrm{REC}}$ of lightpaths, avoiding the deployment of unnecessary amplifiers. The baseline approach, however, places amplifiers at all network nodes, which penalizes the overall cost of amplifiers. Referring to Table $\mathrm{V}$, which shows minimum and average OSNR values for each OA deployment strategy, we can also observe that cost savings by MinOA are achieved while maintaining a comparable performance in terms of OSNR. Specifically, we note a reduction in the case of SP-MinOA-pre of $0.72 \mathrm{~dB}$ in terms of minimum OSNR and $2.06 \mathrm{~dB}$ in terms of average OSNR in comparison to the baseline, i.e., SP-Baseline. Note that, in some cases, as for location-unconstrained ML-MinOA$b s t$ the minimal OSNR and average OSNR values are higher with respect to baseline for $0.97 \mathrm{~dB}$ and $0.53 \mathrm{~dB}$, respectively.

\section{2) Location-Constrained vs. Location-Unconstrained}

Now we focus location-unconstrained vs location-constrained amplifier placement. Fig. 7 shows that unconstrained amplifier deployment permits cost savings up to $8 \%$ for the baseline and between $2 \%$ and $5 \%$ for the various versions of MinOA. Moreover, as shown in Table $\mathrm{V}$, the unconstrained deployment provides advantages also in terms of minimum and average lightpaths OSNR. Specifically, in case of SP-MinOA, locationunconstrained deployment reaches $0.29 \mathrm{~dB}$ higher minimal OSNR compared to location-constrained and in case of $M L$ MinOA-pre, location-unconstrained reaches $1.34 \mathrm{~dB}$ higher average OSNR compared to location-constrained. The OSNR improvement is due to the fact that, with the locationunconstrained deployment, MinOA places amplifiers with lower gain ranges, hence, compared to the location-constrained case, less ASE noise is introduced.

\section{3) Effect of the Routing Algorithm: SP vs. ML}

Now we focus on the effect of the routing algorithm on the OA placement. Overall, $S P$ and $M L$ routing algorithms show comparable results in terms of OA cost, but, considering OSNR (see Table V), $M L$ routing provides significantly better performance compared to $S P$ routing. This is because $M L$ routing assumes paths with minimum loss (in case no amplifiers are pre-deployed) or paths with highest compensation of loss (in case amplifiers are pre-deployed) thus achieving overall better minimum and average OSNR values.

Table VI. Spectral occupation (in \#FSUS) for each routing algorithm

\begin{tabular}{|c|c|c|c|}
\hline Routing algorithm & SP & ML & ML pre-deployed \\
\hline Total number of FSUS & 2817 & 2226 & 2289 \\
\hline
\end{tabular}

Now, we observe the spectrum occupation for each routing algorithm. Note that the spectrum occupation per demand is calculated as the number of FSUs needed for that demand, times the number of links the demand traverses. For example, a demand carrying $100 \mathrm{Gbps}$, i.e. $3 \mathrm{FSU}$, and traversing 4 links, occupies 12 FSUs in total. The total spectrum occupation is given by the total number of FSUs for all the traffic demands in the network. We note that the two algorithms show different overall spectrum occupation (see Table VI). $M L$ routing utilizes $19 \%$ to $22 \%$ less spectrum with respect to $S P$ routing, because, given the relatively short links length, $M L$ routing tends to accommodate lightpaths on routes with low number of nodes, since they introduce a significant loss, and consequently lower number of links, thus reducing occupied spectrum. Conversely, $S P$ routing focuses on lightpaths length, i.e., it aims at reducing propagation losses only, so it routes lightpaths on paths with high number of links (note that the link lengths are relatively short in metro).

\section{4) Further Analysis on larger topologies}

We now consider only ML routing and location-unconstrained amplifier deployment (the strategy with best results) to extend our analysis on a larger reference network, as shown in Fig. 8 (characteristics shown in Table IV).

Table VII shows the total cost as well as average and minimum OSNR for different deployment strategies adopting $M L$ routing and location-unconstrained OA placement.

Results show that $M L-M i n O A$ achieves $48 \%$ of cost savings with respect to baseline, and 35\% (respectively, 36\%) with respect to ML-MinOA-pre (respectively, ML-MinOA-bst). In terms of OSNR, $M$ inOA performs comparably to Baseline in terms of OSNR with an exception of a penalty of $1.98 \mathrm{~dB}$ in average OSNR for the case of MinOA-pre. 


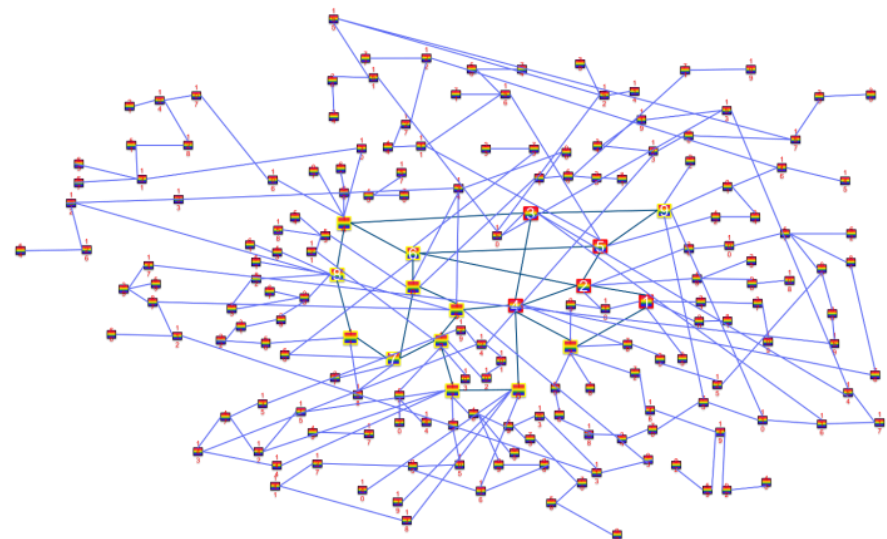

Figure 8. Schematic representation of 159 Node-Metro network topology

This shows that in metro networks, where number of nodes is relatively high, a green-field deployment of amplifiers is largely preferred to significantly reduce the deployment of unnecessary amplifiers and therefore their cost.

Table VII. Cost in $\mathrm{cu}$ and OSNR in dB for 159Node-Metro topology

\begin{tabular}{|c|c|c|}
\hline Strategy & Cost & minOSNR (avgOSNR) \\
\hline ML-MinOA & 466.8 & $13.17(21.45)$ \\
\hline ML-MinOA-pre & 583.6 & $13.30(19.41)$ \\
\hline ML-MinOA-bst & 582.6 & $13.01(21.42)$ \\
\hline ML-Baseline & 909.6 & $13.04(21.39)$ \\
\hline
\end{tabular}

\section{CONCLUSION AND FUTURE WORK}

The OA placement problem is a complex cross-layer non-linear optimization problem. We propose a novel optimization algorithm for the deployment of OAs in metro networks. We consider placement of amplifiers along fiber links and at network nodes. We evaluate the impact of considering different types of OAs, different routing algorithms and OA placement strategies. Numerical results obtained over realistic metro network topologies show that significant savings in total cost of OAs up to $48 \%$ are achieved in comparison to baseline OA placement strategies. Moreover, we also consider the case in which placement of different types of OAs is not constrained to specific locations, achieving further savings in total OA cost and higher ONSR (up to $1.34 \mathrm{~dB}$ ) with respect to the locationconstrained placement. We also observe that minimal-loss routing leads to higher overall OSNR, increasing average OSNR up to $0.31 \mathrm{~dB}$ compared to shortest-path routing.

As future work, we observe that one of the main drawbacks of $\mathrm{MinOA}$ is its greedy nature in placing OAs, i.e., once an OA is placed, its placement is not re-discussed. We are currently investigating evolutionary algorithms, i.e., genetic algorithms, that allow more effective exploration of search space. We are also investigating impact of OA gain ripple in this problem. of Optical Communications and Networking, 7 (12), pp. B184-B193, 2015.

[3] L. Sadeghioon et al., "Software-based burst mode reception implementation for time-domain wavelength interleaved networks", in European Conference on Optical Communication (ECOC), 2015.

[4] P. Poggiolini et al., "The GN-model of fiber non-linear propagation and its applications", in Journal of Lightwave Technology, 32(4), pp.694-721, 2014.

[5] J. Pedro and N. Costa, "Optimized Hybrid Raman/EDFA Amplifier Placement for DWDM Mesh Networks," in Journal of Lightwave Technology, 36(9), pp. 1552-1561, 2018.

[6] M. Cantono et al., "Networking benefit of hybrid fiber amplification for lightpath regenerators saving," Optical Fiber Communications Conference and Exhibition (OFC), 2017.

[7] A. Ferrari, M. Cantono, U. Waheed, A. Ahmad and V. Curri, "Networking benefits of advanced DSP techniques and hybrid fiber amplification," International Conference on Transparent Optical Networks (ICTON), 2017.

[8] A. Ferrari, A. Tanzi, S. Piciaccia, G. Galimberti and V. Curri, "Selection of Amplifier Upgrades Addressed by Quality of Transmission and Routing Space," Optical Fiber Communications Conference and Exposition (OFC), 2019.

[9] B. Ramamurthy, J. Iness and B. Mukherjee, "Optimizing amplifier placements in a multiwavelength optical LAN/MAN: the equally powered-wavelengths case," in Journal of Lightwave Technology, 16(9), pp. 1560-1569, 1998.

[10] B. Ramamurthy, J. Iness and B. Mukherjee, "Optimizing amplifier placements in a multiwavelength optical LAN/MAN: the unequally powered wavelengths case," in IEEE/ACM Transactions on Networking, 6(6), pp. 755-767, 1998.

[11] O. Ayoub, M. Ibrahimi, F. Musumeci, A. Castoldi, R. Pastorelli and M. Tornatore, "Routing and Spectrum Assignment with Optical Amplifier Placement in Elastic Metro-Aggregation Networks", European Conference on Optical Communication (ECOC), 2019.

[12] R. Pastorelli, G. Bosco, S. Piciaccia and F. Forghieri, "Network planning strategies for next-generation flexible optical networks [invited]," in IEEE/OSA Journal of Optical Communications and Networking, 7(3), pp. A511-A525, 2015.

[13] P. Poggiolini et al., "The LOGON strategy for low-complexity control plane implementation in new-generation flexible networks," Optical Fiber Communication Conference and Exposition and the National Fiber Optic Engineers Conference (OFC/NFOEC), 2013.

[14] J. Pedro, N. Costa and M. Gunkel "Raman amplification: Key enabler for next-generation flexible optical networks?" International Conference on Transparent Optical Networks (ICTON), 2017

\section{REFERENCES}

[1] C. Tremblay et al., "Agile optical networking: Beyond filtered solutions" Optical Fiber Communications Conference and Exposition (OFC), 2018.

[2] M. Gunkel et al., "Vendor-interoperable elastic optical interfaces: Standards, experiments, and challenges [Invited]," in IEEE/OSA Journal 